\title{
Developmental linkage between the interpretation of ambiguous referent, Theory of Mind, and perspective-taking.
}

\section{Taro Murakami}

Faculty of humanities, Department of Human Development, Kyusyu Women’s University, Japan, taro.village@gmail.com

\section{Introduction}

- In verbal communication, a receiver often needs to resolve referential ambiguity.

-Reference Assignment task (Murakami \& Hashiya, 2014)

What's this? $\begin{gathered}\text { What's } \\ \text { about this? }\end{gathered}$

- The ability to refer referents appropriately has been found to develop between 3- and 5-year-old, however 3-year-old children fail to disambiguation based on the context (Murakai \& Hashiya, 2014).

- To examine the cognitive mechanisms,

-Cognitive shift (Dimensional change card-sorting task) (Murakami \& Hashiya, 2014)

Phonological Loop (the forward digit span task)

(Meng, Murakami, \& Hashiya, 2017)

$\rightarrow$ These performances have been found to be unrelated to performance on assignment to ambiguous questions.

-And the difficulty of disambiguation was not due to local correspondence such as "this"-referent mappings in a particular context (Murakami \& Hashiya, 2019).

In order to interpret the utterance appropriately, what social cognitive abilities are behind this phenomenon?

\section{The aim of this study:}

To examine the developmental linkage between the abilities

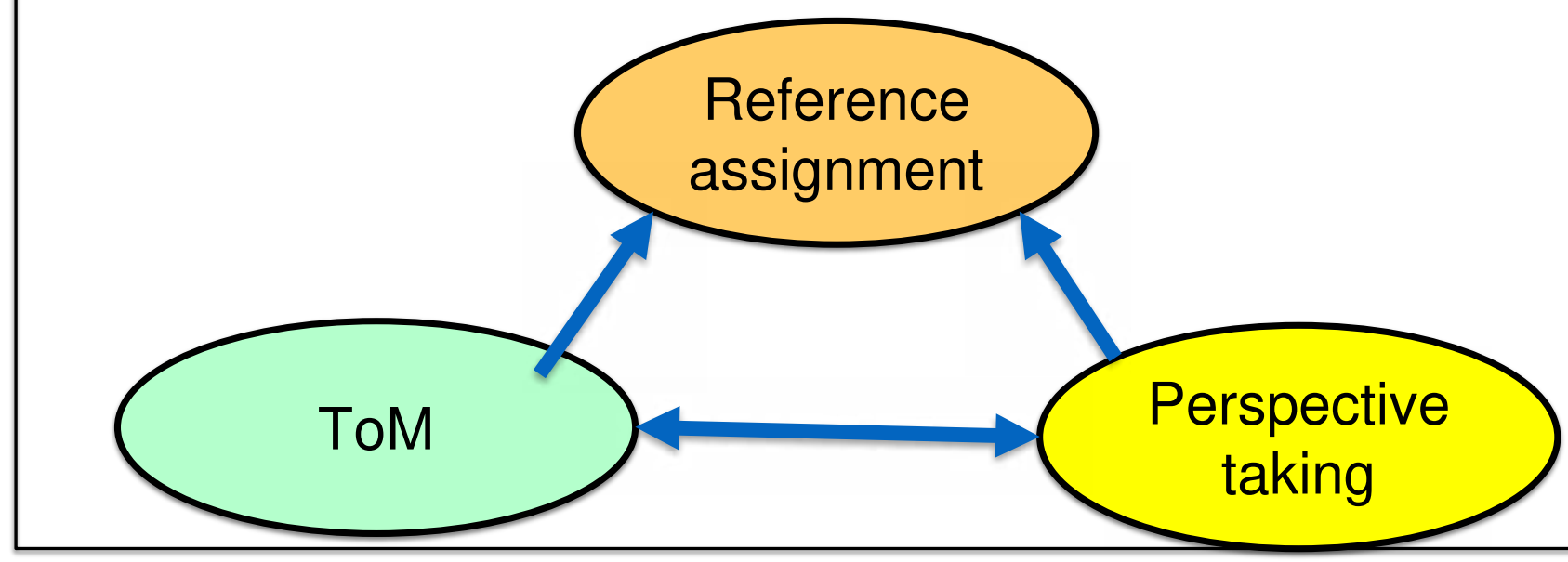

\section{Method}

Participants: 17 3-4 year old children (7 female, $M=49.8$ months, $S D=5.7$ )

25 5-6 year old children (10 female, $M=67.4$ months, $S D=5.1$ )

-Reference assignment task (Murakami \& Hashiya, 2014)

Stimulus: Illustrations (umbrella, shoe, chair, cup, or car) $\times$ (red, blue, yellow, or green) Instructions: Explicit Question "What's this?" / "What's the color of this?" Implicit Question "What's about this?"

Reassignment score: the number of the trials that children answered appropriately in both questions (RA score: 0 -4)

The order of the trials (name/color) was counterbalanced across participants.

- Referential communication task (Nielsen \& Graham, 2009)

Materials: A wooden display case $(52 \mathrm{~cm} \times 52 \mathrm{~cm})$, with nine smaller boxes (each $17 \mathrm{~cm} \times 17 \mathrm{~cm})$.

The side of the display case facing the child was open while the other side (facing experimenter) had sliding doors. The sliding doors were used to conceal one object from experimenter's view.

Instructions: (e.g.) "Pick up the duck" $\rightarrow$ target object / referential alternative (score: 0-5)

False Belief task

- unexpected transfer task (Wimmer \& Perner, 1983) • unexpected contents task (Hogrefe et al., 1986)

$\rightarrow$ ToM score: $0-2$

\section{Results and Discussion}

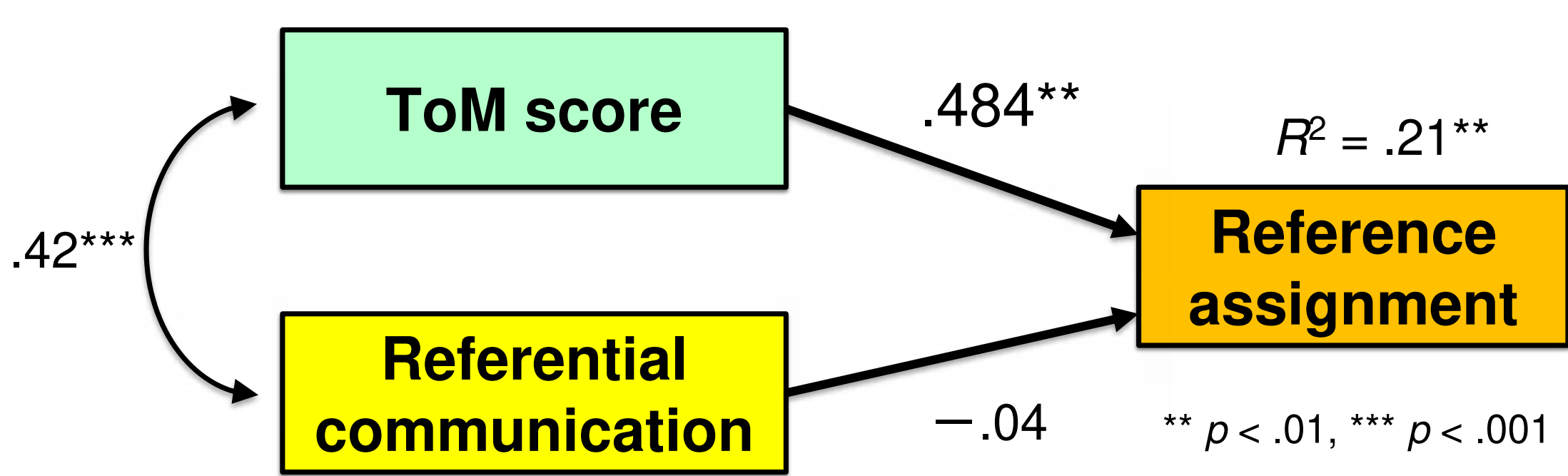

-The ability to disambiguate the ambiguous referent related to the understanding of others' false belief.

- Pragmatic interpretation as a form of "mind-reading" in linguistic communication might be similar to the link between "theory of mind" abilities and linguistic complementation (de Villiers, 2007; Low, 2010).

However, contrary to expectations, the score of the referential communication task did not affect the score of the reference assignment, in spite of the tasks measuring disambiguation.

-Further study is needed to clarify the cognitive mechanisms behind pragmatics. 